\title{
Telmisartan suppresses cardiac hypertrophy by inhibiting cardiomyocyte apoptosis via the NFAT/ANP/BNP signaling pathway
}

\author{
XIURONG LI ${ }^{1}$, YUHUAI LAN ${ }^{2}$, YAN WANG ${ }^{1}$, MINGHAO NIE ${ }^{1}$, YANHONG LU ${ }^{1}$ and ERYANG ZHAO ${ }^{3}$ \\ ${ }^{1}$ Department of Pathology, Heilongjiang Provincial Hospital; \\ ${ }^{2}$ Intensive Care Unit, Heilongjiang Provincial Hospital; ${ }^{3}$ Department of Oral Pathology, \\ Stomatological Hospital, Harbin Medical University, Harbin, Heilongjiang 150036, P.R. China
}

Received December 18, 2015; Accepted January 17, 2017

DOI: $10.3892 / \mathrm{mmr} .2017 .6318$

\begin{abstract}
Telmisartan, a type of angiotensin II (Ang II) receptor inhibitor, is a common agent used to treat hypertension in the clinic. Hypertension increases cardiac afterload and promotes cardiac hypertrophy. However, the ventricular Ang II receptor may be activated in the absence of hypertension. Therefore, telmisartan may reduce cardiac hypertrophy by indirectly ameliorating hypertensive symptoms and directly inhibiting the cardiac Ang II receptor. Nuclear factor of activated T-cells (NFAT) contributes to cardiac hypertrophy via nuclear translocation, which induces a cascade of atrial natriuretic peptide (ANP) and brain/B-type natriuretic peptide (BNP) expression and cardiomyocyte apoptosis. However, NFAT-mediated inhibition of cardiac hypertrophy by telmisartan remains poorly understood. The present study demonstrated that telmisartan suppressed cardiomyocyte hypertrophy in a mouse model of cardiac afterload and in cultured cardiomyocytes by inhibiting NFAT nuclear translocation, as well as by inhibiting ANP and BNP expression and cardiomyocyte apoptosis, in a dose-dependent manner. The present study provides a novel insight into the potential underlying mechanisms of telmisartan-induced inhibition of cardiomyocyte hypertrophy, which involves inhibition of NFAT activation, nuclear translocation and the ANP/BNP cascade.
\end{abstract}

\section{Introduction}

Heart failure follows hypertension as the end stage of cardiac hypertrophy, and is associated with high mortality rates

Correspondence to: Dr Eryang Zhao, Department of Oral Pathology, Stomatological Hospital, Harbin Medical University, 143 Yiman Street, Harbin, Heilongjiang 150036, P.R. China E-mail: mashengli1996@163.com

Key words: telmisartan, cardiomyocyte hypertrophy, nuclear factor of activated T-cells nuclear translocation, atrial natriuretic peptide, apoptosis worldwide. Therefore, reducing hypertension may ameliorate cardiac hypertrophy and consequently prevent heart failure (1). The renin-angiotensin system serves an important role in maintaining normal blood pressure, as well as the development of hypertension and secondary hypertensive organ disorders (2). Angiotensin II (Ang II) receptor blockers (ARB), known as sartans, have been widely used to treat hypertension in the clinic. Telmisartan is a well-known nonpeptide ARB that selectively inhibits Ang II receptor type 1 (AT1) without affecting additional receptor systems $(3,4)$. A significant number of reports have demonstrated that telmisartan may be involved in reducing systolic and diastolic blood pressure (BP) (5-7). In addition, telmisartan has been reported to enhance renal blood flow, thereby increasing renal function $(8,9)$. In diabetic and nondiabetic patients, telmisartan may improve insulin sensitivity, reduce weight gain and prevent hepatic steatosis by regulating caloric expenditure and lipid metabolism (10-12). In addition, telmisartan may penetrate the blood-brain barrier in a dose- and time-dependent manner and prevent cognitive decline $(13,14)$.

As well as reducing BP, a previous study demonstrated that telmisartan may suppress cardiac hypertrophy in TGR (mREN2)27 transgenic rats; a rat model of fulminant hypertension (15). Telmisartan monotherapy significantly reduced left atrial volume, alleviating left ventricular hypertrophy (16). In the rat myocardial infarction model, recovery of left ventricular function was improved with telmisartan administration, indicating that telmisartan may prevent unfavorable cardiac remodeling via the reduction of cardiac hypertrophy and fibrosis (17). However, it is thought that the effect of telmisartan on different organs may be mediated by inhibition of the AT1 receptor (18).

Cardiac hypertrophy is characterized by cell enlargement which involves physiological and pathological hypertrophy (19). Pathological cardiac hypertrophy is often coupled with interstitial and perivascular fibrosis, as well as apoptosis and the release of atrial natriuretic peptides (ANP) and brain/B-type natriuretic peptides (BNP). Upon initiation of cardiac hypertrophy, concentric hypertrophy is the primary phenotype that resists high afterload, and is known as the adaptive phase. As cardiac damage progresses, cell length increases, which 
leads to increased hypertrophy (20). In cardiac hypertrophy, nuclear factor of activated T-cells (NFAT) is considered to be an important mediator of a number of signal-transduction pathways involved in the coordination of pathological stimulation (21). In addition, NFAT may be stimulated by the AT1 receptor (22). However, it is unknown whether the effect of telmisartan on the cardiomyocyte AT1 receptor blockade may extend to inactivation of the NFAT pathway.

The aim of the present study was to clarify whether telmisartan prevents cardiomyocyte hypertrophy by inhibiting NFAT nuclear translocation, ANP/BNP release and cardiomyocyte apoptosis.

\section{Materials and methods}

Animals. A total of 23 male C57/BL6 mice (age 8-10 weeks; weight 20-23 g) were used for the purposes of this study. All mice were purchased from Heze Better Biotechnology Co., Ltd. (Shangdong, China) and had free access to normal chow diet and water, temperature and humidity were kept at $22-24^{\circ} \mathrm{C}$, $40-60 \%$ with a $12 \mathrm{~h}$ light/12 h dark cycle. Mice were divided into 4 groups (5-7 mice per cage) with the same average body weight. Out of these, two groups were used for sham (control group) operations with the administration of either saline $(n=5)$ or telmisartan $(n=5)$. Aortic binding (AB) operations were performed on mice in the remaining two groups, together with the administration of either saline $(n=6)$ or telmisartan $(n=7)$. All mice were sacrificed at 4 weeks post-AB operation.

$A B$ model. The afterload-induced pressure model was generated by $\mathrm{AB}$ under abdominal anesthesia as described previously (23). Briefly, the chest was first opened, and $A B$ of the aortic arch between the brachiocephalic artery and left common carotid artery was performed using a 27-gauge needle as the standard binding level to lead to the aortic arch narrow. Following successful binding, the needle was removed and the chest cavity was closed. The same operation without $\mathrm{AB}$ was performed on mice in the sham group.

Neonatal rat cardiomyocyte primary culture. Wistar rats (age, 1-3 days) were purchased from the Experimental Animal Center of the Academy of Military Medical Sciences (Beijing, China). Hearts were harvested under aseptic conditions on a clean bench. Following 3 washes with sterilized phosphate-buffered saline to remove excess blood, the hearts were minced, washed with Hank's Balanced Salt Solution at $4^{\circ} \mathrm{C}$ and digested in cardiomyocyte balance suspension liquid containing $0.1 \%$ trypsin and $0.025 \%$ collagenase (Sigma-Aldrich; Merck KGaA; Darmstadt, Germany) for $15 \mathrm{~min}$. The undigested heart tissue was re-digested 4 or 5 times. Following complete digestion, cardiomyocytes were separated and isolated by centrifugation using the Percoll gradient system (upper, $45.3 \%$ percoll; bottom, 58.5\% percoll; Sigma-Aldrich; Merck KGaA) as described previously (24). The separated rat cardiomyocytes were cultured in Dulbecco's modified Eagle's medium containing $10 \%$ fetal bovine serum and penicillin $(0.2 \%)$ (Sigma-Aldrich; Merck KGaA) at a density of $1 \times 10^{5}$ cells $/ \mathrm{cm}^{2}$ in a $5 \% \mathrm{CO}_{2}$ incubator at $37^{\circ} \mathrm{C}$. At 2 days following isolation, and when cardiomyocyte beating was confirmed, the cells were used for the purposes of downstream experiments. All animal experiments were approved by the Ethics Review Committee of Harbin Medical University (Harbin, China).

Telmisartan in vivo administration and in vitro treatment. Telmisartan (BoehringerIngelheim Shanghai Pharmaceuticals, Co., Ltd., Shanghai, China) was dissolved in saline prior to in vivo administration at a dose of $50 \mathrm{mg} / \mathrm{kg} / \mathrm{day}$. Saline or telmisartan were administered to the mice once-a-day via oral gavage, commencing 5 days prior to $\mathrm{AB}$ operations and continuing until they were sacrificed at 4 weeks post-AB operation. For the treatment of neonatal primary rat cardiomyocytes in vitro, telmisartan was first dissolved in dimethyl sulfoxide. Three different concentrations of telmisartan (5, 20 and $50 \mu \mathrm{M})$ were used for these experiments. In addition, Ang II (10 $\mu \mathrm{M}$; Sigma-Aldrich; Merck KGaA) was used to induce cardiomyocyte hypertrophy in vitro. Rat cardiomyocytes were treated with the vehicle (dimethyl sulfoxide), telmisartan and Ang II for $12 \mathrm{~h}$.

Morphological and histological analyses. Mouse heart samples were fixed in $4 \%$ formaldehyde overnight at room temperature and embedded in paraffin. Blocks were cut into $5-\mu \mathrm{m}$ thick sections and de-waxed in xylene for $10 \mathrm{~min}$ and stained with hematoxylin and eosin (H\&E) at room temperature. Briefly, the sections were placed in distilled water, stained with alum hematoxylin at room temperature for $10 \mathrm{~min}$, rinsed under running tap water, differentiated with $0.3 \%$ acid alcohol at room temperature for $\sim 30 \mathrm{sec}$, rinsed in running tap water again and rinsed in Scott's tap water substitute (Sigma-Aldrich; Merck $\mathrm{KGaA}$ ) prior to rinsing in tap water again. The sections were subsequently stained with eosin for $2 \mathrm{~min}$. Following that, sections were observed (Olympus FluoView ${ }^{\mathrm{TM}}$ FV1000) to evaluate cardiac morphology and measure cardiomyocyte size. The thickness of the anterior, posterior, lateral and septum heart walls was measured, and the average of these four parameters was calculated as the average wall thickness. Similarly, the internal dimensions (presented as an average of the horizontal and vertical measurements) of left ventricles were measured and an average value was calculated as average internal dimensions. To investigate cardiomyocyte size, $\sim 50$ cardiomyocytes stained by $H \& E$ were measured and the average value was calculated as the average area of myocytes per sample. All of these measurements were performed using ImageJ software (v1.48; National Institutes of Health, Bethesda, MD, USA). Heart weight was measured by an experimental scale.

TUNEL assay. TUNEL assay was performed on heart sections $(5-\mu \mathrm{m})$ with a TdT-FragEL DNA Fragmentation Detection kit to quantify apoptosis. Counterstaining with fluorescence mounting medium containing DAPI (blue; Thermo Fisher Scientific, Inc., Waltham, MA, USA) was performed to visualize normal nuclei. Sections were observed by use of a fluorescence microscope Olympus FluoView ${ }^{\mathrm{TM}}$ FV1000. Measurements of the apoptotic nuclei percentage were obtained from the border zone area of sections from each heart and 8 fields were randomly selected from the border zone of each heart and the average TUNEL-positive percentage (\%) was calculated.

Immunocytochemical analysis of NFATc3 nuclear translocation. Cardiomyocytes were collected and cultured 
on glass bottom coverslips in 4-well dishes that had been previously coated with collagen II, as described above for rat cardiomyocyte primary culture. Following treatment with Ang II or telmisartan, cells were fixed with $10 \%$ acetone at $4^{\circ} \mathrm{C}$ for $20 \mathrm{~min}$. After the glass slides were washed by TBS with $0.5 \%$ Tween-20, 3 times, double immunofluorescent staining was performed using a specific antibody against NFATc3 (cat. no. sc-8405, 1:500; Santa Cruz Biotechnoloy, Inc., Dallas, TX, USA) at room temperature for $2 \mathrm{~h}$, followed by the addition of a donkey anti-rabbit IgG (red; Alexa Fluor ${ }^{\circledR}$ 488; cat. no. ab150077; 1:500; Abcam, Cambridge, UK) for $1 \mathrm{~h}$ at room temperature. Finally, following 3 washes with TBS, slides were mounted in fluorescence mounting medium containing DAPI (blue; Thermo Fisher Scientific, Inc.). Cells were observed by use of a fluorescence microscope, Olympus FluoView ${ }^{\mathrm{TM}} \mathrm{FV} 1000 .{ }^{\circ} \mathrm{C}$.

Reverse transcription-quantitative polymerase chain reaction $(P C R)$. Total RNA was extracted from the mouse hearts using TRIzol (Invitrogen; Thermo Fisher Scientific, Inc.), and total RNA (2,000 ng) was used, cDNA (50 ng/ $\mu \mathrm{l})$ was synthesized using oligo (dT) primers and the Transcriptor First Strand cDNA Synthesis kit (cat. no. 04896866001; Roche Applied Science, Penzberg, Germany). PCR amplifications were performed using LightCycler ${ }^{\circledR} 480$ SYBR-Green I Master (cat. no. 04887352001; Roche Applied Science). Target gene expression levels were normalized to the expression of the gene encoding the $18 \mathrm{~S}$ ribosomal RNA subunit by the $\Delta \Delta \mathrm{Cq}$ method, as described (25). The primers used were as follows: Myosin heavy chain $\alpha$ isoform (Myh6), forward, 5'-AGA TCATCAAGGCCAAGGCA-3', and reverse, 5'-CGCTGG GTGGTGAAATCATT-3'; GATA binding protein 4 (Gata4), forward, 5'-AGCTCCGTGTCCCAGACG-3', and reverse, 5'-TCTGTGGAGACTGGCTGACG-3'; collagen I, forward, 5'-ATGTTCAGCTTTGTGGACCTC-3', and reverse, 5'-TCC CTCGACTCCTACATCTTC-3'; collagen III, forward, 5'-TGG TTTCTTCTCACCСТTCTTC-3', and reverse, 5'-TGCATC CCAATTCATCTACGT-3'.

Western blot analysis. Mouse ventricular cytoplasmic protein was extracted using lysis buffer (50 mM Tris- $\mathrm{HCl}, \mathrm{pH} 7.5$, containing $150 \mathrm{mM} \mathrm{NaCl}, 25 \mathrm{mM}$ EDTA, $0.25 \%$ sodium deoxycholate, and $1 \mathrm{mM}$ dithiothreitol). The nuclear protein precipitate was extracted using radioimmunoprecipitation assay (RIPA) buffer (50 mM Tris-HCl, pH 7.5, containing $150 \mathrm{mM}$ $\mathrm{NaCl}, 1 \mathrm{mM}$ EDTA and 1\% NP-40). Total protein was extracted from cultured cells using RIPA lysis buffer containing $2 \%$ phenylmethylsulfonyl fluoride, $10 \%$ complete protease inhibitor cocktail, 10\% PhosSTOP (Sigma-Aldrich; Merck KGaA), $5 \% \mathrm{NaF}$ and $1 \% \mathrm{Na}_{3} \mathrm{VO}_{4}$. The protein concentration was determined using a Pierce BCA Protein assay kit (cat. no. 23225; Pierce; Thermo Fisher Scientific, Inc.). Proteins (20 $\mu \mathrm{g})$ were electrophoresed by 10\% SDS-PAGE (cat. no. NP0301BOX; Invitrogen; Thermo Fisher Scientific, Inc.) and subsequently electrotransferred to a polyvinylidene difluoride membrane (cat. no. IPVH00010; Merck Millipore). Membranes were blocked in TBS with $0.5 \%$ Tween-20 containing 5\% skim milk for $60 \mathrm{~min}$ at room temperature. Membranes were subsequently incubated with the following primary antibodies overnight at $4^{\circ} \mathrm{C}$ : NFATc3 (cat. no. sc-8405; 1:1,000), NFATc4 (cat. no. sc-13036; 1:1,000), ANP (cat. no. sc-20158; 1:2,000), BNP (cat. no. sc-67455; 1:2,000), caspase 3 (cat. no. sc-7148; 1:1,000), GAPDH (cat. no. sc-365062; 1:2,000) and lamin (cat. no. sc-20680; 1:2,000). All primary antibodies were purchased from Santa Cruz Biotechnology, Inc. The membrane was incubated at room temperature for $1 \mathrm{~h}$ with Peroxidase-affiniPure goat anti-mouse IgG (H+L; cat. no. 115-035-003; 1:2,000; Jackson ImmunoResearch Laboratories, Inc., West Grove, PA, USA) or Peroxidase-affiniPure goat anti-rabbit IgG $(\mathrm{H}+\mathrm{L}$; cat. no. 111-035-003; 1:2,000; Jackson ImmunoResearch Laboratories, Inc.). A FluorChem E (Cell Biosciences Pty, Ltd., Heidelberg, VIC, Australia) imaging system was used to visualize the signals. GAPDH was used as a loading control.

Statistical analysis. Results are expressed as the mean \pm standard deviation. Comparisons between 2 groups were analyzed using a Student's t-test. Comparisons among $>2$ groups were analyzed using one-way analysis of variance and Turkey's multiple comparisons test. Statistical analysis was performed using GraphPad Prism (v6.0; GraphPad Software, Inc., La Jolla, CA, USA) $\mathrm{P}<0.05$ was considered to indicate a statistically significant difference.

\section{Results}

Telmisartan inhibited cardiac hypertrophy. In order to determine whether telmisartan inhibits cardiac hypertrophy, an in vivo study using a mouse $\mathrm{AB}$ model was performed. At 4 weeks following the $\mathrm{AB}$ operation, mice administered with saline exhibited a hypertrophied heart with increased wall thickness, an enlarged left ventricular dimension and increased heart weight $(\mathrm{HW})$ /body weight (BW) ratio, when compared with the saline-treated sham group (Fig. 1A, $\mathrm{C}$ and $\mathrm{E})$. By contrast, mice administered with telmisartan exhibited reduced cardiac hypertrophy, with a significantly reduced wall thickness, smaller left ventricular dimensions and a lower HW/BW ratio when compared with the saline-treated AB group (Fig. 1A, C and E). In addition, cardiomyocyte size was significantly lower in the hearts of mice in the telmisartan-treated group when compared to that of the saline-treated AB group (Fig. 1B and D). The mRNA expression levels of cardiac hypertrophy markers, Myh6 and Gata4, as well as the markers of cardiac fibrosis, collagen I and collagen III were increased when compared with the sham telmisartan-treated AB group, but at a significantly lower level when compared with the saline-treated AB group (Fig. 1F and G). These results indicated that telmisartan may inhibit cardiac hypertrophy and fibrosis in a mouse model of cardiac afterload.

Telmisartan inhibited NFAT nuclear translocation and $A N P / B N P$ expression. NFAT is an important nuclear transcriptional factor involved in cardiac hypertrophy (26). Thus, the authors investigated whether telmisartan inhibited NFAT nuclear translocation. There are five subtypes of NFAT in cardiomyocytes, however, it is thought that NFATc3 and NFATc4 are the most highly expressed in cardiomyocytes, and therefore NFATc3 and NFATc4 may be the two most important subtypes (27). Following the extraction of cardiomyocyte nuclear protein, the expression levels of NFATc3 and NFATc4 

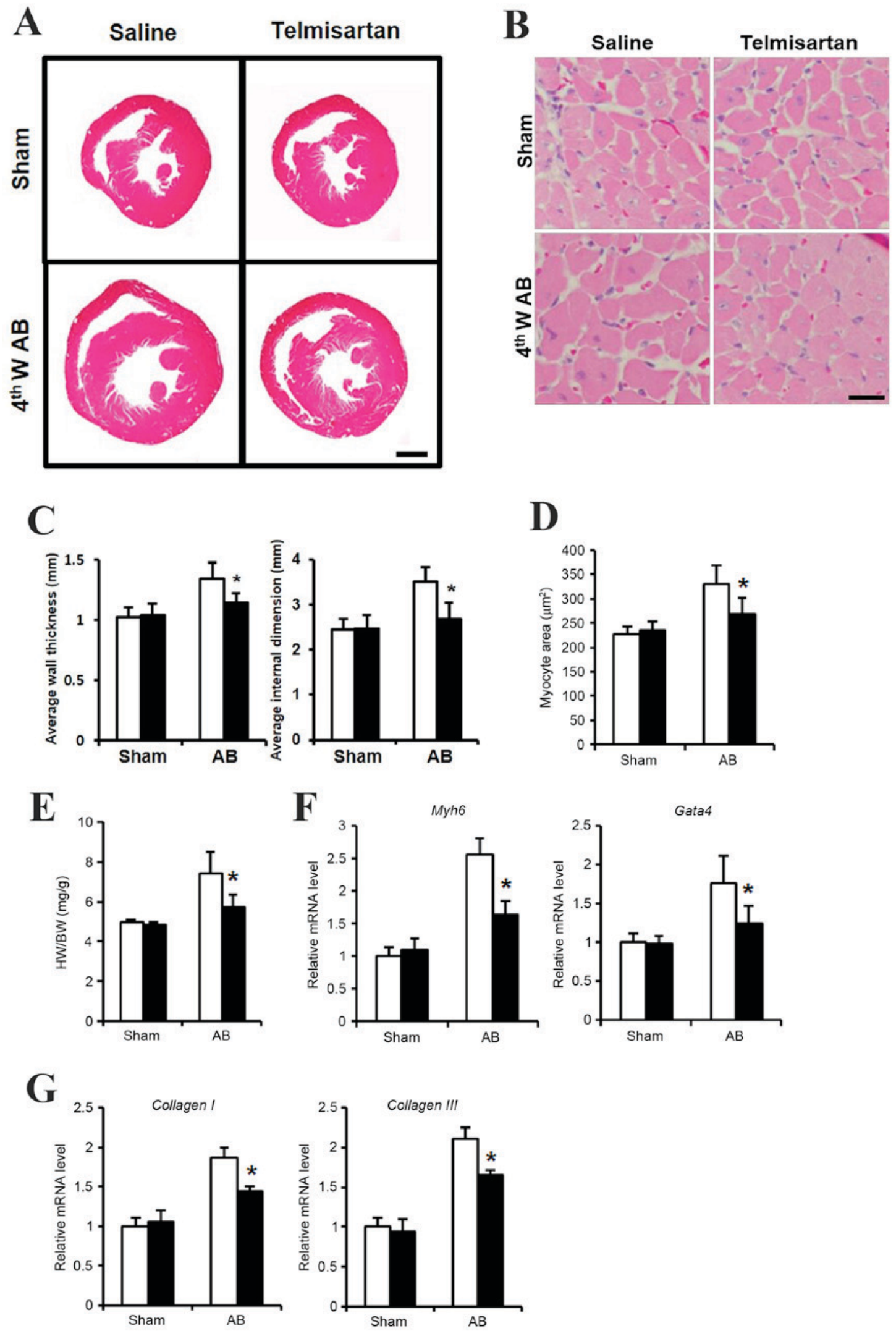

Figure 1. Cardiac hypertrophy phenotype of telmisartan treatment in a mouse AB model. (A) H\&E staining of whole hypertrophic hearts at 4 weeks following $\mathrm{AB}$ operation $(\mathrm{n}=5-7$; scale bar, $1 \mathrm{~mm}$ ). (B) H\&E staining sections were analyzed further to observe cellular hypertrophy at 4 weeks following the AB operation (n=5; scale bar, $25 \mu \mathrm{m}$ ). (C) Average heart wall thickness (left panel) and average left ventricular internal dimension (right panel) according to the H\&E staining images. (D) Quantification of hypertrophied cardiomyocyte size. (E) The HW/BW (heart weight/body weight) ratio as a cardiac hypertrophy index. The mRNA levels of (F) Myh6 and Gata4 as markers of cardiac hypertrophy, and (G) collagen I and collagen III as markers of cardiac fibrosis, as determined by reverse transcription-quantitative polymerase chain reaction. Data are presented as the mean \pm standard deviation. " $\mathrm{P}<0.05$ vs. the saline-treated $\mathrm{AB}$ group. $\mathrm{AB}$, aortic binding; $\mathrm{H} \& \mathrm{E}$, hematoxylin and eosin staining; 4thW AB, 4 weeks post-AB operation; HW, heart weight; BW, body weight; Myh6, myosin heavy chain a isoform; GATA4, GATA binding protein 4. 

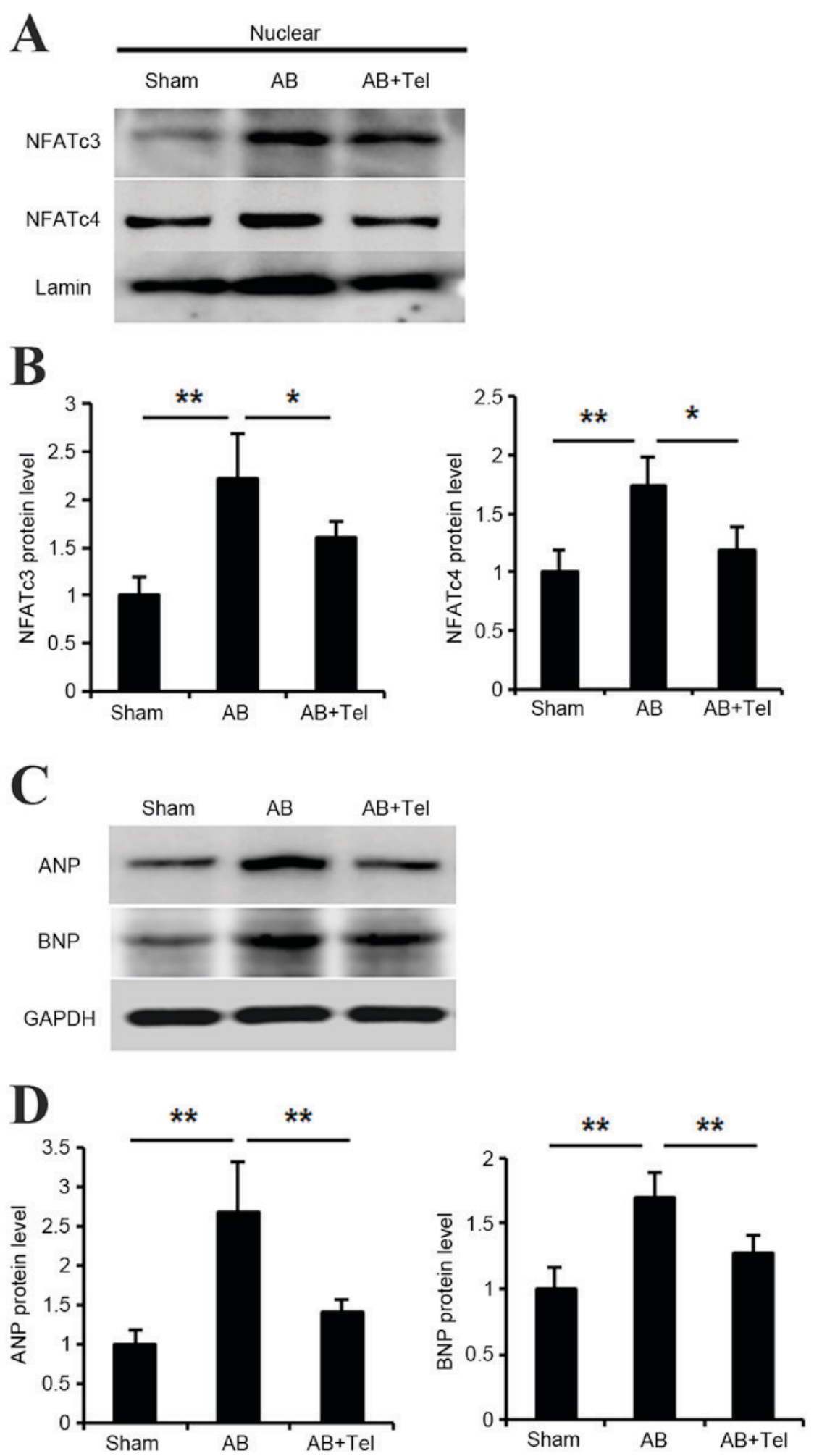

Figure 2. Cardiac signaling pathway responses to Tel administration as determined by western blot analysis. (A) Nuclear NFATc3 and NFATc4 protein expression levels in heart lysates and (B) quantification of data. (C) Cardiac ANP and BNP protein expression levels and (D) quantification of data. $n=3$ per group and experiments were performed independently three times. Data are presented as the mean \pm standard deviation. ${ }^{*} \mathrm{P}<0.05$ and ${ }^{* *} \mathrm{P}<0.01$ as indicated. Tel, telmisartan; NFAT, nuclear factor of activated T-cells; ANP, atrial natriuretic peptide; BNP, brain/B-type natriuretic peptide.

were significantly increased in the $\mathrm{AB}$ group when compared with the sham group $(\mathrm{P}<0.01$; Fig. $2 \mathrm{~A}$ and $\mathrm{B})$. However, this expression was significantly reduced in the telmisartan-treated $\mathrm{AB}$ group when compared with the untreated $\mathrm{AB}$ group $(\mathrm{P}<0.05)$ (Fig. 2A and $\mathrm{B})$.
Activated NFAT has been reported to stimulate the expression of ANP and BNP $(28,29)$. Thus, the present study investigated whether ANP/BNP expression may be inhibited by telmisartan treatment. ANP and BNP expression significantly increased in the hearts of mice from the $A B$ group 


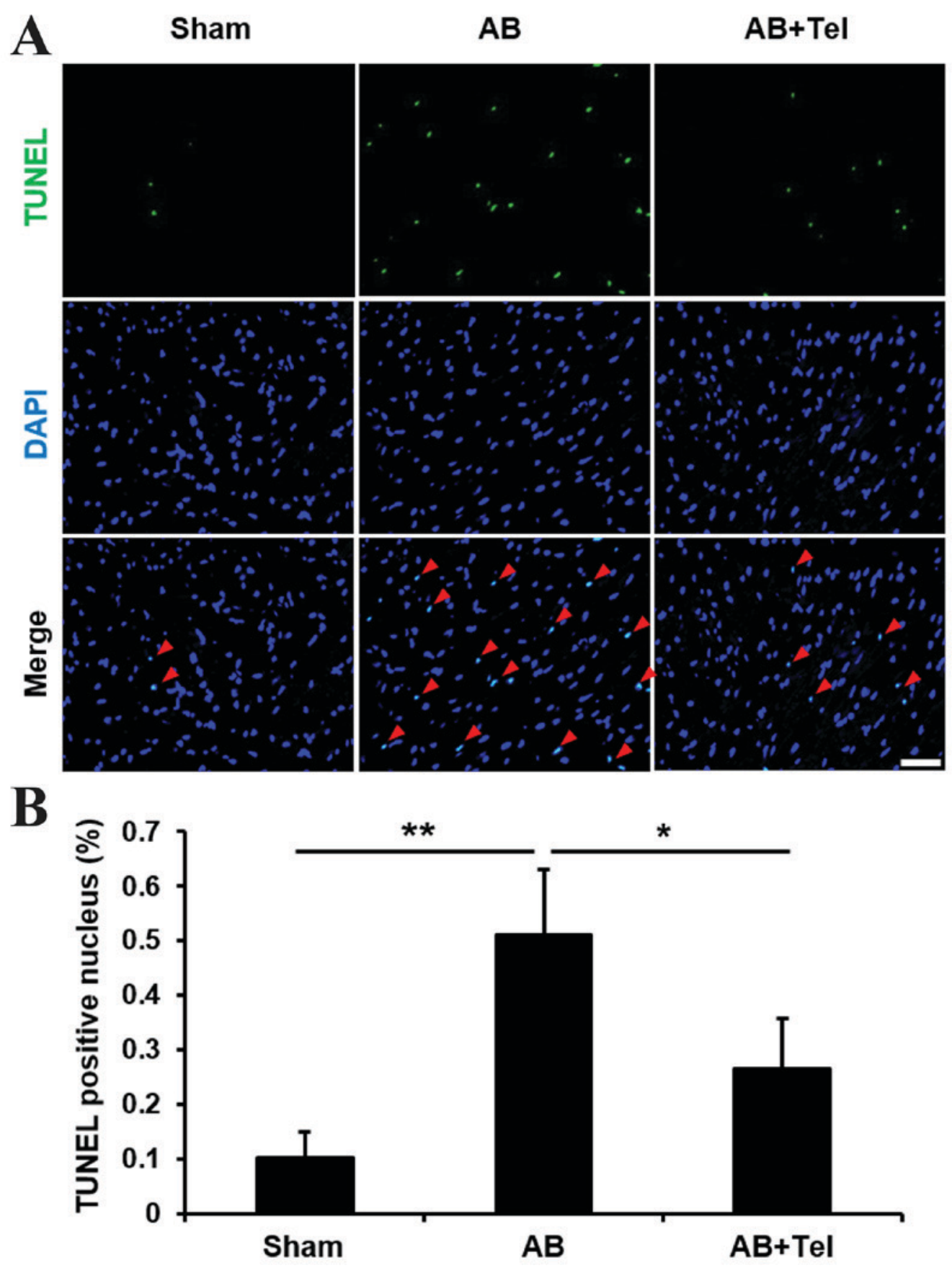

Figure 3. Detection of apoptosis in cardiac myocytes treated with Tel by TUNEL and DAPI staining. (A) TUNEL staining (green) indicates cardiomyocyte apoptosis, and DAPI staining (blue) indicates cardiomyocyte nuclei. Merged TUNEL and DAPI staining images demonstrate apoptotic cardiomyocyte nuclei, which are indicated by red arrows (scale bar, $50 \mu \mathrm{m}$ ). (B) Percentage of TUNEL-positive nuclei in each experimental group. Data are presented as the mean \pm standard deviation $(\mathrm{n}=5) .{ }^{*} \mathrm{P}<0.05$ and ${ }^{* *} \mathrm{P}<0.01$ as indicated. Tel, telmisartan; TUNEL, terminal deoxynucleotidyl transferase dUTP nick end labeling; $\mathrm{AB}$, aortic binding.

when compared with the sham group ( $\mathrm{P}<0.01$; Fig. $2 \mathrm{C}$ and $\mathrm{D})$. However, telmisartan administration significantly lowered ANP and BNP levels when compared to the untreated AB group $(\mathrm{P}<0.01$; Fig. $2 \mathrm{C}$ and $\mathrm{D})$. The inhibitory effect of telmisartan on ANP expression was marginally more pronounced than its effect on BNP protein levels (Fig. 2C and D).

Telmisartan inhibited cardiac apoptosis. As ANP and BNP reportedly induce apoptosis $(30,31)$, and cardiac afterload may induce damaging levels of apoptosis in heart tissues (32), the anti-apoptotic effects of telmisartan were investigated in the present study. The terminal deoxynucleotidyl transferase dUTP nick end labeling (TUNEL) stain revealed that the percentage of TUNEL-positive nuclei induced by cardiac afterload were significantly reduced by telmisartan administration when compared with the untreated $\mathrm{AB}$ group $(\mathrm{P}<0.05$; Fig. 3). These results indicate that telmisartan may inhibit NFAT nuclear translocation, ANP and BNP expression and cardiomyocyte apoptosis to protect against cardiac afterload-induced cardiac hypertrophy.

Telmisartan suppressed cardiomyocyte hypertrophy, NFAT nuclear translocation, ANP expression and cardiomyocyte 
$\mathbf{A}$
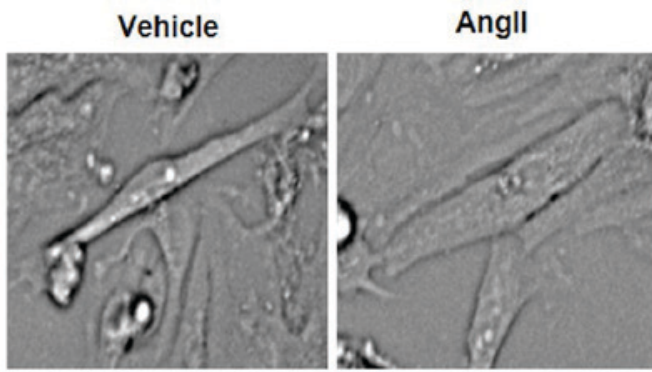

B
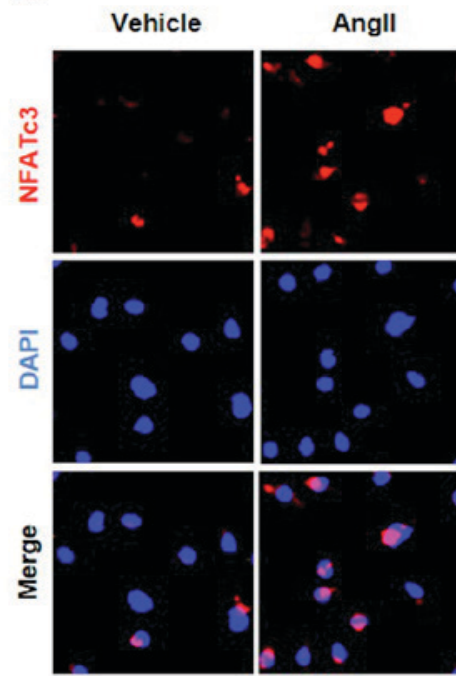

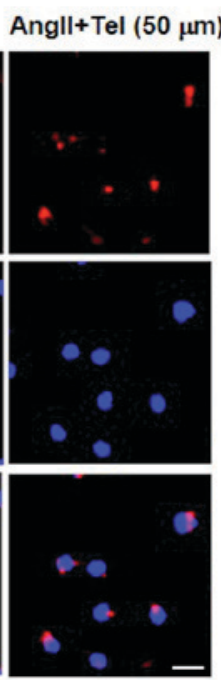

Angll+Tel $(50 \mu \mathrm{m})$

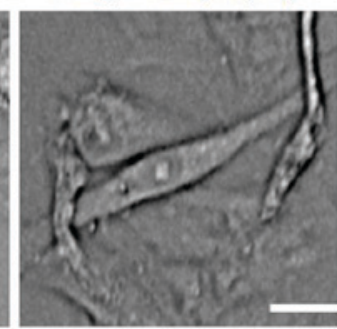

C

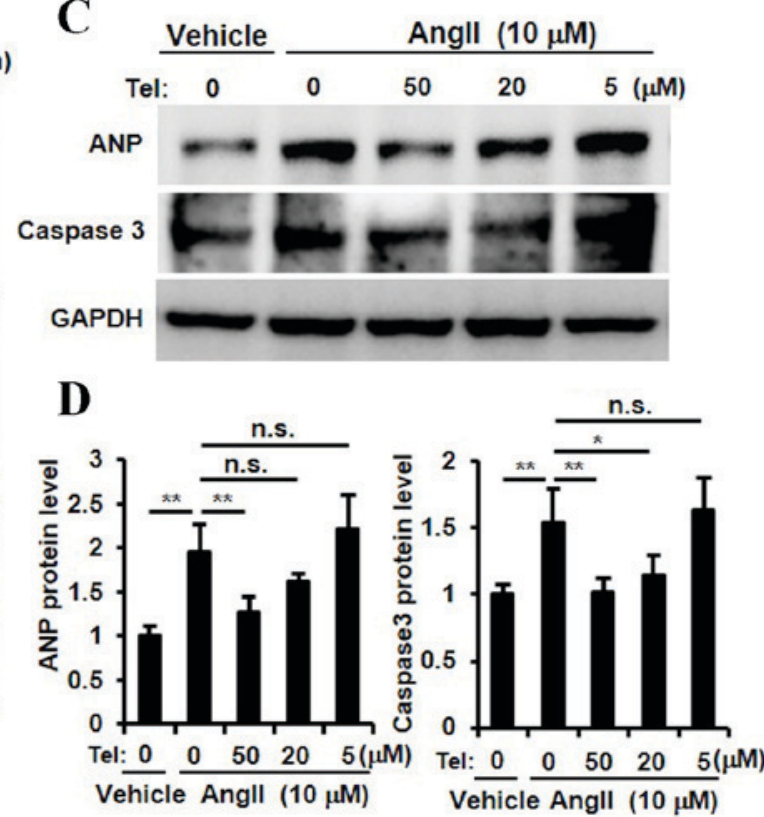

Figure 4. Cardiomyocyte signaling pathway analysis and the phenotypic response to Tel stimulation in a primary neonatal rat cardiomyocyte culture. (A) Primary cardiomyocyte size in response to Ang II and Ang II + Tel stimulation (scale bar, $10 \mu \mathrm{m}$ ). (B) Detection of NFAT nuclear translocation by NFATc3 immunocytochemical and DAPI staining following Ang II + Tel stimulation (scale bar, $10 \mu \mathrm{m}$ ). (C) Western blot analysis of ANP and caspase 3 protein expression following treatment with different doses of Tel $(0,5,20$ and $50 \mu \mathrm{M})$ and (D) quantification of the results. Experiments were performed three times using independent samples $(\mathrm{n}=4)$. Data are presented as mean \pm standard deviation. ${ }^{*} \mathrm{P}<0.05$ and ${ }^{* *} \mathrm{P}<0.01$ as indicated. Tel, telmisartan; Ang II, angiotensin II; NFAT, nuclear factor of activated T-cells; ANP, atrial natriuretic peptide; n.s. not significant.

apoptosis in vitro. The results presented so far provide evidence to suggest that telmisartan may inhibit cardiac hypertrophy, NFATc3 and NFATc4 nuclear translocation, ANP expression and cardiac cell apoptosis in a mouse model of cardiac afterload. In order to investigate whether these biochemical effects are detectable in cardiomyocytes directly, a primary neonatal rat cardiomyocyte culture was established and stimulated with Ang II. As shown in Fig. 4A, telmisartan appeared to inhibit Ang II-induced cardiomyocyte hypertrophy, which was consistent with the results observed in the in vivo mouse AB model (Fig. 1A-E). In order to determine whether telmisartan may inhibit the nuclear translocation of NFAT, ANP expression and cardiomyocyte apoptosis, cardiomyocytes were stimulated with three different concentrations of telmis$\operatorname{artan}(5,10$ and $50 \mu \mathrm{m})$. A high concentration of telmisartan $(50 \mu \mathrm{m})$ inhibited Ang II-induced NFATc3 nuclear translocation (Fig. 4B). In addition, ANP expression was significantly inhibited by telmisartan in a dose-dependent manner $(\mathrm{P}<0.01$, $50 \mu \mathrm{M}$ vs. $0 \mu \mathrm{M}$ telmisartan; Fig. $4 \mathrm{C}$ and D). Furthermore, the Ang II-stimulated increase in caspase 3 protein expression levels, which is a known marker for apoptosis (33), was inhibited by telmisartan in a dose-dependent manner $(\mathrm{P}<0.01$, $50 \mu \mathrm{M}$ vs. $0 \mu \mathrm{M}$ telmisartan; Fig. 4C and D). These results indicated that telmisartan may inhibit NFAT nuclear translocation, ANP expression, Ang II-induced cardiomyocyte apoptosis and suppress cardiomyocyte hypertrophy in vitro.

\section{Discussion}

Angiotensin-converting enzyme inhibitors (ACEIs) and ARBs are important agents in the treatment of hypertension, however, $\sim 20 \%$ of patients tolerate ARBs but are unable tolerate ACEIs (34). This indicates that ARBs may be more effective for broader clinical use. In a previous study involving the AB mouse cardiac hypertrophy model, the AT1 receptor was activated without the involvement of Ang II (35). Candesartan, olmesartan and Losartan were reported to inhibit pressure overload-induced cardiac hypertrophy even in the absence of Ang II (36), and telmisartan directly inhibited cardiomyocyte hypertrophy in primary rat cardiomyocyte cultures (37). These reports indicate that telmisartan may serve an effective role in inhibiting cardiac hypertrophy. 
Although NFAT has been reported as a universal factor for the induction of cardiac hypertrophy, it is unclear whether NFAT activation may be involved in the suppression of cardiac hypertrophy by telmisartan. In the present study, telmisartan inhibited cardiomyocyte hypertrophy in a mouse model of cardiac afterload, with a reduction in cardiomyocyte size and reduced expression of cardiomyocyte hypertrophy and cardiac fibrosis markers, which is consistent with previous reports (38). However, it was previously reported that telmisartan does not exert its inhibitory effects on cardiomyocyte hypertrophy following 2 weeks of administration in the absence of Ang II (36), which implies that telmisartan may require additional time to exert its inhibitory effects.

NFAT is thought to be important for inducing cardiomyocyte hypertrophy (39). The authors of the present study therefore hypothesized that telmisartan may inhibit NFAT activation. The results demonstrated that telmisartan inhibited the cardiac overload-induced activation of NFATc3 and NFATc4. Previous studies have demonstrated that extracellular signal-regulated kinases (Erk) 1/2 and NFAT form a complex in cardiomyocytes. Erk1/2 directly regulate NFAT DNA binding activation, and exert their effects on NFAT synergy without increasing NFAT translocation and translation. Erk1/2 and NFAT are together required to induce cardiac hypertrophy $(40,41)$. Although the inhibition of NFAT nuclear translocation by telmisartan was only investigated in the present study, it remains formally possible that Erk1/2 and NFAT form a complex to exert their cardiomyocyte hypertrophy-inducing effects. The inhibition of NFAT nuclear translocation may be dependent on Erk1/2 inhibition, however, the present study did not investigate this possibility. Nevertheless, the Erk1/2-NFAT complex may additionally be inhibited by telmisartan.

Previous studies have revealed that peroxisome proliferator-activated receptor- $\gamma$ (PPAR $\gamma)$ activation may be involved in the underlying mechanisms of telmisartan-induced inhibition of cardiomyocyte hypertrophy (42), and PPAR $\gamma$ and its ligand may further inhibit the nuclear translocation of NFAT (43). These studies indicate that telmisartan-induced inhibition of NFAT nuclear translocation may be dependent on telmisartan-induced PPARg activation.

NFAT has been observed to participate in pathological cardiac hypertrophy (39), and activated NFAT promotes ANP or BNP release and induces cell apoptosis $(41,44)$. The present study demonstrated that telmisartan inhibited ANP/BNP expression and apoptosis in the heart and cardiomyocytes. Notably, telmisartan did not inhibit BNP expression as effectively as ANP expression. Therefore, it is possible that an additional pathway involving NFAT and BNP exists. Alternatively, telmisartan may have inhibited NFAT as well as an additional complex involving NFAT.

Following this investigation there are two points that require further investigation in future studies. Firstly, whether telmisartan inhibits the Erk1/2 and NFAT complex, and secondly, whether the telmisartan-mediated inhibition of NFAT nuclear translocation is dependent on PPARg activation.

In conclusion, the present study demonstrated that telmisartan suppressed cardiomyocyte hypertrophy in vivo and in vitro, potentially by suppressing cardiomyocyte ANP/BNP expression and apoptosis, which may be dependent on the inhibition of NFAT nuclear translocation. These results may provide a novel insight into the mechanism of telmisartan-induced cardiomyocyte hypertrophy inhibition.

\section{References}

1. Frohlich ED, Apstein C, Chobanian AV, Devereux RB, Dustan HP, Dzau V, Fauad-Tarazi F, Horan MJ, Marcus M, Massie B, et al: The heart in hypertension. N Engl J Med 327: 998-1008, 1992.

2. Curtiss C, Cohn JN, Vrobel T and Franciosa JA: Role of the renin-angiotensin system in the systemic vasoconstriction of chronic congestive heart failure. Circulation 58: 763-770, 1978.

3. McClellan KJ and Markham A: Telmisartan. Drugs 56: 1039-1046, 1998

4. Wienen W, Hauel N, Van Meel JC, Narr B, Ries U and Entzeroth M: Pharmacological characterization of the novel nonpeptide angiotensin II receptor antagonist, BIBR 277. Br J Pharmacol 110: 245-252, 1993.

5. Amerena J,Pappas S, OuelletJP, Williams L and O'Shaughnessy D: ABPM comparison of the anti-hypertensive profiles of telmisartan and enalapril in patients with mild-to-moderate essential hypertension. J Int Med Res 30: 543-552, 2002.

6. Saha L: Comparison of the efficacy and tolerability of telmisartan and enalapril in patients of mild to moderate essential hypertension. Indian J Pharmacol 43: 360, 2011.

7. Neutel JM, Littlejohn TW, Chrysant SG and Singh A; Telmisartan Study Group: Telmisartan/Hydrochlorothiazide in comparison with losartan/hydrochlorothiazide in managing patients with mild-to-moderate hypertension. Hypertens Res 28: 555-563, 2005.

8. Wienen W and Entzeroth M: Effects on binding characteristics and renal function of the novel, non-peptide angiotensin II antagonist BIBR277 in the rat. J Hypertens 12: 119-128, 1994.

9. Makino H, Haneda M, Babazono T, Moriya T, Ito S, Iwamoto Y, Kawamori R, Takeuchi M and Katayama S; INNOVATION Study Group: Prevention of transition from incipient to overt nephropathy with telmisartan in patients with type 2 diabetes. Diabetes Care 30: 1577-1578, 2007.

10. Derosa G, Ragonesi PD, Mugellini A, Ciccarelli L and Fogari R: Effects of telmisartan compared with eprosartan on blood pressure control, glucose metabolism and lipid profile in hypertensive, type 2 diabetic patients: A randomized, double-blind, placebo-controlled 12-month study. Hypertens Res 27: 457-464, 2004.

11. Honjo S, Nichi Y, Wada Y, Hamamoto Y and Koshiyama H: Possible beneficial effect of telmisartan on glycemic control in diabetic subjects. Diabetes Care 28: 498, 2005.

12. Nagel JM, Tietz AB, Göke B and Parhofer KG: The effect of telmisartan on glucose and lipid metabolism in nondiabetic, insulin-resistant subjects. Metabolism 55: 1149-1154, 2006.

13. Gohlke P, Weiss S, Jansen A, Wienen W, Stangier J, Rascher W, Culman J and Unger T: AT1 receptor antagonist telmisartan administered peripherally inhibits central responses to angiotensin II in conscious rats. J Pharmacol Exp Ther 298: 62-70, 2001.

14. Mogi M, Li JM, Tsukuda K, Iwanami J, Min LJ, Sakata A, Fujita T, Iwai M and Horiuchi M: Telmisartan prevented cognitive decline partly due to PPAR-gamma activation. Biochem Biophys Res Commun 375: 446-449, 2008.

15. Böhm M, Lippoldt A, Wienen W, Ganten D and Bader M: Reduction of cardiac hypertrophy in TGR(mREN2)27 by angiotensin II receptor blockade. Mol Cell Biochem 163-164: 217-221, 1996.

16. Mattioli AV, Zennaro M, Bonatti S, Bonetti L and Mattioli G: Regression of left ventricular hypertrophy and improvement of diastolic function in hypertensive patients treated with telmisartan. Int J Cardiol 97: 383-388, 2004.

17. Maejima Y, Okada H, Haraguchi G, Onai Y, Kosuge H, Suzuki J and Isobe M: Telmisartan, a unique ARB, improves left ventricular remodeling of infarcted heart by activating PPAR gamma. Lab Invest 91: 932-944, 2011.

18. Siragy H: Angiotensin II receptor blockers: Review of the binding characteristics. Am J Cardiol 84: 3S-8S, 1999.

19. D'Ascenzi F, Pelliccia A, Corrado D, Cameli M, Curci V, Alvino F, Natali BM, Focardi M, Bonifazi M and Mondillo S: Right ventricular remodelling induced by exercise training in competitive athletes. Eur Heart J Cardiovasc Imaging 17: 301-307, 2016. 
20. Bernardo BC, Weeks KL, Pretorius L and McMullen JR: Molecular distinction between physiological and pathological cardiac hypertrophy: Experimental findings and therapeutic strategies. Pharmacol Ther 128: 191-227, 2010.

21. Heineke J and Molkentin JD: Regulation of cardiac hypertrophy by intracellular signalling pathways. Nat Rev Mol Cell Biol 7: 589-600, 2006.

22. Irani RA, Zhang Y, Blackwell SC, Zhou CC, Ramin SM, Kellems RE and Xia Y: The detrimental role of angiotensin receptor agonistic autoantibodies in intrauterine growth restriction seen in preeclampsia. J Exp Med 206: 2809-2822, 2009.

23. Rockman HA, Ross RS, Harris AN, Knowlton KU Steinhelper ME, Field LJ, Ross J Jr and Chien KR: Segregation of atrial-specific and inducible expression of an atrial natriuretic factor transgene in an in vivo murine model of cardiac hypertrophy. Proc Natl Acad Sci USA 88: 8277-8281, 1991.

24. Chlopcíková S, Psotová J and Miketová P: Neonatal rat cardiomyocytes-a model for the study of morphological, biochemical and electrophysiological characteristics of the heart. Biomed Pap Med Fac Univ Palacky Olomouc Czech Repub 145: 49-55, 2001.

25. Livak KJ and Schmittgen TD: Analysis of relative gene expression data using real-time quantitative PCR and the 2(-Delta Delta C(T)) Method. Methods 25: 402-408, 2001

26. Molkentin JD: Calcineurin-NFAT signaling regulates the cardiac hypertrophic response in coordination with the MAPKs. Cardiovasc Res 63: 467-475, 2004.

27. Pu WT, Ma Q and Izumo S: NFAT transcription factors are critical survival factors that inhibit cardiomyocyte apoptosis during phenylephrine stimulation in vitro. Circ Res 92: 725-731, 2003.

28. Tokudome T, Horio T, Kishimoto I, Soeki T, Mori K, Kawano Y, Kohno M, Garbers DL, Nakao K and Kangawa K: Calcineurin-nuclear factor of activated T cells pathway-dependent cardiac remodeling in mice deficient in guanylyl cyclase A, a receptor for atrial and brain natriuretic peptides. Circulation 111: 3095-3104, 2005.

29. Liang F, Lu S and Gardner DG: Endothelin-dependent and -independent components of strain-activated brain natriuretic peptide gene transcription require extracellular signal regulated kinase and p38 mitogen-activated protein kinase. Hypertension 35: $188-192,2000$.

30. Wu CF, Bishopric NH and Pratt RE: Atrial natriuretic peptide induces apoptosis in neonatal rat cardiac myocytes. J Biol Chem 272: 14860-14866, 1997.

31. Suenobu N, Shichiri M, Iwashina M, Marumo F and Hirata Y: Natriuretic peptides and nitric oxide induce endothelial apoptosis via a cGMP-dependent mechanism. Arterioscler Thromb Vasc Biol 19: 140-146, 1999.

32. Diep QN, El Mabrouk M, Yue P and Schiffrin EL: Effect of AT(1) receptor blockade on cardiac apoptosis in angiotensin II-induced hypertension. Am J Physiol Heart Circ Physiol 282: H1635-H1641, 2002.

33. Porter AG and Jänicke RU: Emerging roles of caspase-3 in apoptosis. Cell Death Differ 6: 99-104, 1999.
34. Telmisartan Randomised AssessmeNt Study in ACE iNtolerant subjects with cardiovascular Disease (TRANSCEND) Investigators, Yusuf S, Teo K, Anderson C, Pogue J, Dyal L, Copland I, Schumacher H, Dagenais G and Sleight P: Effects of the angiotensin-receptor blocker telmisartan on cardiovascular events in high-risk patients intolerant to angiotensin-converting enzyme inhibitors: A randomised controlled trial. Lancet 372: 1174-1183, 2008

35. Zou Y, Akazawa H, Qin Y, Sano M, Takano H, Minamino T, Makita N, Iwanaga K, Zhu W, Kudoh S, et al: Mechanical stress activates angiotensin II type 1 receptor without the involvement of angiotensin II. Nat Cell Biol 6: 499-506, 2004.

36. Li L, Zhou N, Gong H, Wu J, Lin L, Komuro I, Ge J and Zou Y: Comparison of angiotensin II type 1-receptor blockers to regress pressure overload-induced cardiac hypertrophy in mice. Hypertens Res 33: 1289-1297, 2010.

37. Chang WH, Yan JJ, Li X, Guo HY and Liu Y: Effects of telmisartan on angiotensin II-induced cardiomyocyte hypertrophy and p-ERK $K_{1} / 2$ phosphorylation in rat cultured cardiomyocytes. Asian Biomed 5: 459-465, 2011.

38. Muller P, Kazakov A, Semenov A, Jagoda P, Friedrich EB, Böhm M and Laufs U: Ramipril and telmisartan exhibit differential effects in cardiac pressure overload-induced hypertrophy without an additional benefit of the combination of both drugs. J Cardiovasc Pharmacol Ther 18: 87-93, 2013.

39. Wilkins BJ, Dai YS, Bueno OF, Parsons SA, Xu J, Plank DM, Jones F, Kimball TR and Molkentin JD: Calcineurin/NFAT coupling participates in pathological, but not physiological, cardiac hypertrophy. Circ Res 94: 110-118, 2004

40. Sanna B, Bueno OF, Dai YS, Wilkins BJ and Molkentin JD Direct and indirect interactions between calcineurin-NFAT and MEK1-extracellular signal-regulated kinase 1/2 signaling pathways regulate cardiac gene expression and cellular growth. Mol Cell Biol 25: 865-878, 2005.

41. Robbs BK, Lucena PI and Viola JP: The transcription factor NFAT1 induces apoptosis through cooperation with Ras/Raf/MEK/ERK pathway and upregulation of TNF- $\alpha$ expression. Biochim Biophys Acta 1833: 2016-2028, 2013.

42. Yamagishi $\mathrm{S}$ and Takeuchi M: Telmisartan is a promising cardiometabolic sartan due to its unique PPAR-gamma-inducing property. Med Hypotheses 64: 476-478, 2005.

43. Bao Y, Li R, Jiang J, Cai B, Gao J, Le K, Zhang F, Chen S and Liu P: Activation of peroxisome proliferator-activated receptor gamma inhibits endothelin-1-induced cardiac hypertrophy via the calcineurin/NFAT signaling pathway. Mol Cell Biochem 317: 189-196, 2008.

44. Molkentin JD, Lu JR, Antos CL, Markham B, Richardson J, Robbins J, Grant SR and Olson EN: A calcineurin-dependent transcriptional pathway for cardiac hypertrophy. Cell 93: 215-228, 1998 